\title{
¿Cuál es la situación de la Ley de Bosques en la Región Chaqueña a diez años de su sanción? Revisar su pasado para discutir su futuro
}

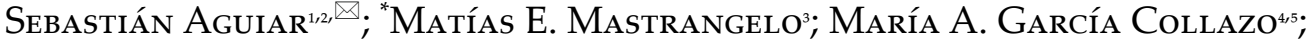 \\ Gonzalo H. Camba Sans ${ }^{1,5}$; Clara E. Mosso ${ }^{6}$; Lucía Ciuffoli ${ }^{4}$; Mariana Schmidt ${ }^{7}$; \\ María Vallejos ${ }^{1,5}$; Lorenzo Langbehn ${ }^{8}$; Miguel Brassiolo ${ }^{14}$; Daniel Cáceres ${ }^{*}$; \\ Gabriela Merlinsky ; José M. Paruelo ${ }^{1,5,13}$; Lucas SeghezzO ${ }^{10}$; Luciana Staiano ${ }^{1,5}$; \\ Marcos Texeira ${ }^{1,5}$; José N. Volante ${ }^{11}$ \& SAntiago R. VeróN ${ }^{12,5}$ \\ ${ }^{1}$ Laboratorio de Análisis Regional y Teledetección (IFEVA-CONICET), Facultad de Agronomía, Universidad de Buenos Aires \\ (FAUBA). ${ }^{2}$ Cátedra de Ecología (FAUBA). ${ }^{3}$ Grupo de Agroecosistemas y Paisajes Rurales, Unidad Integrada Balcarce (INTA \\ y Facultad de Ciencias Agrarias, UNMDP). ${ }^{4}$ Dirección Nacional de Bosques, Ministerio de Ambiente y Desarrollo Sustentable \\ de la Nación. ${ }^{5}$ Departamento de Métodos Cuantitativos y Sistemas de Información (FAUBA). ${ }^{6}$ Licenciatura en Ciencias \\ Ambientales (FAUBA). ${ }^{7}$ Grupo de Estudios Ambientales, Instituto de Investigaciones Gino Germani, Facultad de Ciencias \\ Sociales, Universidad de Buenos Aires-CONICET. ${ }^{8}$ Facultad de Humanidades, Ciencias Sociales y de la Salud, Universidad \\ Nacional de Santiago del Estero (UNSE). ${ }^{9}$ Facultad de Ciencias Agropecuarias, Universidad Nacional de Córdoba-CONICET. \\ ${ }^{10}$ Instituto de Investigaciones en Energía No Convencional (CONICET), Universidad Nacional de Salta. ${ }^{11}$ Laboratorio de \\ Teledetección y SIG, Estación Experimental Agropecuaria Salta (INTA). ${ }^{12}$ Instituto de Clima y Agua $\left(\right.$ INTA); ${ }^{13}$ Instituto \\ nacional de investigaciones agropecuarias. INIA La Estanzuela.Uruguay. ${ }^{14}$ Facultad de Ciencias Forestales (UNSE). ${ }^{*}$ Ex Aequo
}

Resumen. En un complejo escenario ambiental, productivo y socioeconómico, el 28 de noviembre de 2007 fue sancionada en Argentina la Ley Nacional N 26.331 de "Presupuestos Mínimos de Protección Ambiental de los Bosques Nativos" (conocida como "Ley de bosques") con el propósito de proteger los bosques nativos a escala nacional. En este artículo nos proponemos realizar una síntesis crítica de la información disponible acerca de esta ley a diez años de su sanción, con una aproximación que toma en cuenta aspectos ambientales, económicos y sociales. Caracterizamos el desempeño de esta ley en la Región Chaqueña en cuanto a diferentes dimensiones, identificamos sus principales desafíos y describimos una serie de propuestas que desde el sector de Ciencia y Técnica pueden contribuir a su (re)diseño e implementación en el contexto de las actualizaciones de los Ordenamientos Territoriales de Bosques Nativos provinciales. Para ello, integramos información disponible proveniente de distintas fuentes, tales como normativas (nacionales y provinciales), literatura científica, informes de organismos estatales y de ONG y artículos periodísticos. La Ley de Bosques instaló en la opinión pública de nuestro país la problemática vinculada a la pérdida de bosques nativos y se ha posicionado como el principal instrumento de política forestal nacional para su protección. Si bien hubo una reducción en las tasas de deforestación en la región Chaqueña, no existen evidencias certeras de que esta reducción se deba a su aplicación. La Ley de Bosques en la Región Chaqueña presenta una serie de desafíos para mejorar su desempeño en cuanto a su efectividad, equidad y legitimidad social. En este trabajo se presentan diez observaciones que emergen de la revisión realizada. Por otro lado, se esbozan una serie de propuestas de investigación y acción en torno a la ley vinculadas a esas observaciones.

[Palabras clave: bosques nativos, Chaco, Argentina, ordenamiento territorial, zonificación, OTBN, efectividad, equidad, legitimidad]

\begin{abstract}
Aвstract. What is the status of the Forest Law in the Chaco Region ten years after its enaction? Reviewing its past to discuss its future. In a complex environmental, productive and socioeconomic scenario, on November 28th, 2007, the National Act N²6.331 of "Minimum Standards for the Environmental Protection of Native Forests" (known as the "Forest Law") was sanctioned in Argentina with the purpose of protecting native forests at the national scale. In this article, we aim to critically synthesize the available information about this law ten years after its sanction from an approach that takes into account environmental, economic and social aspects. In particular, we characterize the performance of this law in the Chaco Region in different dimensions, identify its main challenges and describe a series of proposals that from the science and technology sector can contribute to its (re)design and implementation in the context of the revisions of the Provincial Native Forest Land Use Planning Programs. In order to accomplish these objectives, we integrated information available from different sources, such as laws and regulations (national and provincial), scientific literature, reports from government agencies and NGOs and newspaper articles. The Forest Law installed in the public opinion of our country the problems related to the loss of native forests and has positioned itself as the main national instrument for forest conservation. Although deforestation rates lowered in the Chaco Region, there is no clear evidence that this reduction was due to its application. The Forest Law in the Chaco Region presents a series of challenges to improve its performance in terms of its effectiveness, equity and social legitimacy. In this paper, we present ten observations that emerge from the review carried out. On the other hand, linked to these observations, we outline a series of research and action proposals for enhancing the performance of the law.
\end{abstract}

[Keywords: native forest, Chaco, Argentina, land use planning, zonation, effectiveness, equity, legitimacy]

Editor asociado: Esteban Jobbágy 


\section{INTRODUCCIÓN}

Uno de los mayores desafíos que enfrentan los países en desarrollo es resolver el conflicto entre la producción de alimentos y la conservación de la naturaleza (Rudel and Meyfroidt 2014). Esto es particularmente importante en Latinoamérica, pues allí se localizan extensas áreas con potencial para la expansión de la agricultura en el futuro (Lambin et al. 2013). A su vez, también se encuentran importantes reservorios de carbono (West et al. 2010), una elevada diversidad biológica y cultural (Myers et al. 2000; Hoekstra et al. 2005; Balvanera et al. 2012) y una gran proporción de población rural en situación de pobreza crónica (Vakis et al. 2015; Graeub et al. 2016). Frente a este desafío han surgido múltiples alternativas de gobernanza ambiental propuestos por, entre otros, el Estado, organismos internacionales (e.g., ONU), la sociedad civil, ONG (Lambin et al. 2014) y diversos actores privados (empresas, bancos). Entre estas alternativas o instrumentos de gestión se encuentran el ordenamiento territorial (Paruelo et al. 2014), los pagos por servicios ecosistémicos (Engel et al. 2008), las moratorias y las certificaciones (Lambin et al. 2014) y diversas combinaciones de las anteriores. Describir la efectividad de estas alternativas para resolver los compromisos y conflictos que surgen en torno al uso del territorio es una necesidad imperiosa. Además de la efectividad, existen otras dimensiones o principios sobre las cuales es necesario analizar el desempeño de los instrumentos para la gobernanza del uso de la tierra, tales como la eficiencia, la equidad, la legitimidad y la flexibilidad (Adger et al. 2005). Aunque la mayoría de los instrumentos de gestión no promuevan explícitamente estos principios, todos ellos son prerrequisitos indispensables para la efectividad a largo plazo cuando el objetivo es la sustentabilidad del territorio (Boyce 2002).

Los incrementos en la demanda de alimentos, fibras y energía en los países desarrollados y emergentes están siendo abastecidos a través de la expansión o la intensificación de la agricultura en regiones tropicales y subtropicales de países en desarrollo (Gibbs et al. 2010). Desde comienzos del siglo XXI, el Gran Chaco Sudamericano es una de las regiones del mundo donde más se han expandido la agricultura y la ganadería, lo cual hace de esta región un hotspot global de deforestación (Hansen et al. 2013). En esta región, los bosques secos son reemplazados por cultivos anuales (principalmente soja y maíz) y pasturas megatérmicas para alimentar ganado vacuno (Grau et al. 2005). Hasta el año 2012, en la porción argentina y semiárida de esta región, el Chaco Seco argentino, se habían transformado 10.5 millones de hectáreas, lo que representa el $22 \%$ de sus ecosistemas naturales (bosques secos, arbustales, sabanas y pastizales) (Vallejos et al. 2015). La mayor proporción de esta transformación ocurrió durante los últimos quince años y se concentró principalmente en las provincias de Chaco, Salta y Santiago del Estero, las cuales explican el 10, 12 y 23\% del área total transformada, respectivamente (Vallejos et al. 2015).

Los cambios en el uso de la tierra en el Chaco Secoargentinotuvieron diversas consecuencias ambientales y sociales negativas. La conversión de bosques determinó una importante emisión de $\mathrm{CO} 2$, equivalente a $75 \%$ de las emisiones de carbono provenientes de la deforestación en el norte argentino (Gasparri et al. 2008). A su vez, esta conversión estuvo relacionada con un ascenso de la napa freática debido a una menor evapotransipiración de los cultivos respecto al bosque, lo que podría incrementar el riesgo de salinización del suelo (Jobbágy et al. 2008; Amdan et al. 2013; Giménez et al. 2015). La conversión de ecosistemas naturales también afectó la diversidad de aves (Macchi et al. 2013; Mastrangelo and Gavin 2014), la viabilidad de poblaciones de depredadores tope (Quiroga et al. 2014, 2016) y también de otros mamíferos de menor tamaño (Periago et al. 2014). Esto llevó a algunos autores a sugerir que el Chaco Seco argentino está atravesando un proceso de defaunación y un "síndrome de bosque vacío" (Periago et al. 2014).

En cuanto a los impactos socio-económicos, gran parte del avance de la frontera agropecuaria en el Chaco Seco argentino no ocurrió en tierras despobladas, sino en tierras de uso tradicional de comunidades indígenas y campesinas (en algunos casos denominados o autodenominados criollos), cuya tenencia de la tierra en general no es segura. Por lo cual, dicha expansión ha reactivado los históricos conflictos por la tenencia de la tierra (León et al. 1985; Schmidt 2012; Seghezzo et al. 2011, 2017), los cuales podrían enmarcarse bajo el concepto o fenómeno de "acumulación por desposesión" (Cáceres 2015; Harvey 2004). En muchos casos, este fenómeno se encuentra asociado al acaparamiento de tierras (i.e., land grabbing), es decir, el proceso mediante el cual empresas privadas, fondos de inversión o gobiernos adquieren, arriendan o hacen uso de concesiones de tierras en gran escala para 
la extracción de recursos naturales (Fairhead et al. 2014). En la Argentina, y en particular en la Región Chaqueña, este proceso es llevado adelante por empresarios nacionales e internacionales quienes adquieren tierras ocupadas por comunidades que generalmente carecen de títulos de propiedad (Constantino 2016; Goldfarb et al. 2016). En consecuencia, los cambios en el uso, control y apropiación del territorio y los bienes de la naturaleza, se asociaron con incesantes disputas territoriales en la Región Chaqueña. En algunos casos, estas disputas derivaron en situaciones de elevada conflictividad social (de Dios 2006; Aguiar et al. 2016). Las disputas se dirimieron de diferentes maneras (e.g., juicios, acuerdos extrajudiciales y uso ilegal de las fuerzas de seguridad) (Seghezzo et al. 2011) y dieron lugar a diversos procesos de organización entre los actores sociales afectados (Barbetta 2005; Domínguez et al. 2006; Mioni et al. 2013).

En este complejo escenario ambiental, productivo y socioeconómico, el 28 de noviembre de 2007 fue sancionada en la Argentina la Ley Nacional № 26.331 de "Presupuestos Mínimos de Protección Ambiental de los Bosques Nativos" (conocida como "Ley de Bosques") con el propósito de proteger a los bosques nativos a escala nacional. La norma fue impulsada principalmente por algunas de las grandes ONG ambientalistas que operan en el país (Fundación Vida Silvestre, Greenpeace, Fundación Ambiente y Recursos Naturales). Estas ONG lograron reclutar el apoyo masivo de un público predominantemente urbano identificado con la agenda conservacionista, que se materializó en la recolección de más de un millón de firmas a favor del proyecto de ley. También adhirieron al proyecto algunas organizaciones indígenas y campesinas y organismos (gubernamentales y no-gubernamentales, partidos políticos) que cooperan con ellas, ya que para estos actores, la regulación de la deforestación constituía un objetivo estratégico en las luchas por el territorio (Schmidt and Langbehn 2016; Seghezzo et al. 2011). Esta Ley cuenta con dos instrumentos principales. Por un lado, el Ordenamiento Territorial de Bosques Nativos (de aquí en más, el "OTBN"), que es "la norma que zonifica territorialmente el área de bosques nativos existentes en cada jurisdicción de acuerdo a las diferentes categorías de conservación basada en los criterios de sostenibilidad ambiental": Categoría I, áreas de muy alto valor de conservación que deben mantenerse como bosques, Categoría II, áreas de valor medio a alto de conservación, donde el aprovechamiento es posible pero no el desmonte y Categoría III, área de bajo valor de conservación que pueden ser transformadas en su totalidad (Ley 26.331 2009). Por otro lado, el Fondo Nacional para el Enriquecimiento y la Conservación de los Bosques Nativos (de aquí en más, el Fondo), el cual es ejecutado por la Autoridad Nacional de Aplicación y tiene como objetivo "compensar a las jurisdicciones que conservan los bosques nativos, por los servicios ambientales que éstos brindan" (Ley 26.3312009 ).

La sanción de la Ley 26.331 (de aquí en más referida como la "Ley") no estuvo exenta de conflictos. Coaliciones formadas por asociaciones empresariales agropecuarias de las provincias de la Región Chaqueña, algunos gobiernos provinciales de esa región y otros actores que respaldan la lógica económica que impulsa el agronegocio, se opusieron a la Ley argumentando que implicaba un freno al desarrollo para una región que recién se iniciaban en el camino del "progreso" a partir de la incorporación de tierras a la producción agropecuaria (Langbehn 2016, 2015; Schmidt 2013, 2014; Seghezzo et al. 2011). En definitiva, la sanción e implementación de la Ley se desarrolló de modos diversos y mostró diferentes grados de conflictividad, dependiendo de factores tales como las características ambientales, la situación económico-productiva, el mapa de actores en juego (empresarios, diferentes niveles de gobierno, ONG nacionales y locales, movimientos sociales, etc.), las relaciones de poder entre ellos y las alianzas construidas en pos de lograr sus objetivos (Schmidt 2013; Seghezzo et al. 2011).

A más de una década del inicio de los debates parlamentarios en torno al rol y destino de los bosques nativos en la Argentina, consideramos necesario y oportuno realizar un balance de los logros y limitaciones del diseño e implementación de la Ley. A su vez, la Ley indica que los OTBN provinciales deben ser actualizados cada cinco años. Actualmente, se encuentran en marcha estos procesos de actualización, por lo cual creemos necesario identificar los principales desafíos que enfrenta actualmente la Ley en la Región Chaqueña. Para ello, en este artículo nos proponemos realizar una síntesis crítica de la información disponible acerca de la Ley, caracterizar su desempeño, identificar sus principales desafíos y describir una serie de propuestas que desde el sector de Ciencia y 
Técnica pueden contribuir a su (re)diseño e implementación en el contexto de las actualizaciones de los OTBN provinciales. En particular, describiremos diferentes aspectos del diseño e implementación de la Ley desde un enfoque que toma en cuenta tanto la complejidad ambiental como la conflictividad social en la Región Chaqueña. Esta región abarca la totalidad de las provincias de Formosa, Chaco y Santiago del Estero y parte de Salta, Córdoba, Santa Fe, San Luis, Jujuy, Catamarca y La Rioja. Integramos información disponible proveniente de distintas fuentes, tales como normativas (nacionales y provinciales), literatura científica, informes de ONG y de organismos estatales, y artículos periodísticos. Los objetivos específicos del presente trabajo son: a) revisar en forma crítica el desempeño de la Ley en cuanto a su diseño e implementación en diferentes dimensiones e identificar sus principales desafíos, b) identificar vacíos de información y conocimiento a ser cubiertos desde el sector científico-técnico para contribuir al cumplimiento de los objetivos de la Ley, y c) proponer líneas de acción futuras a modo de disparador de la discusión sobre el rol de la interfaz investigación-gestión en los (re)diseños de la Ley.

A continuación, presentamos en forma sintética las diez principales observaciones de la revisión. Cada una de ellas está resumida en una sentencia breve, seguida de párrafos que presentan la evidencia que la respalda. Luego, presentamos una serie de propuestas que sugieren direcciones futuras en la investigación y acción en torno a la Ley, las que se desprenden de las observaciones precedentes.

\section{Diez observaciones A DIEZ AÑos DE LA SANCIÓN DE LA LEY}

1. La Ley instaló en la opinión pública de nuestro país la problemática vinculada a la pérdida de bosques nativos y de servicios ecosistémicos importantes para la sociedad. A su vez, se posicionó como el instrumento principal de política forestal argentina para proteger a los bosques nativos, y permitió que los daños ambientales sean considerados daños jurídicos.

La sanción de la Ley de Bosques fue el resultado de un conjunto de demandas de actores sociales (ONG, comunidad científica, comunidades indígenas y campesinas, entrelos principales) preocupados y afectados por los perjuicios originados por el reemplazo de los bosques nativos por actividades agropecuarias (Seghezzo et al. 2011). Estos reclamos fueron incorporados en la norma y resultaron en el reconocimiento de los bosques nativos como proveedores de "servicios ambientales" (de aquí en más llamados servicios ecosistémicos) cuyo beneficiario es la sociedad en su conjunto, y de la deforestación como proceso a controlar para no comprometer la provisión de servicios ecosistémicos y culturales (Artículos 3, 4 y 5, Ley 26.331 2009). Para garantizar la conservación de los bosques nativos y de los servicios ecosistémicos asociados, la Ley incorporó un instrumento inédito en la legislación argentina (hasta el momento): el Ordenamiento Territorial Participativo. Para la realización del ordenamiento, la Ley postuló una serie de Criterios de Sustentabilidad Ambiental que incluyen tanto valores ecológicos (i.e., conservación de la biodiversidad, vinculación con otros ambientes, potencial forestal y agropecuario de los bosques, conservación de cuencas hidrográficas), como valores culturales que las comunidades indígenas y campesinas otorgan a los bosques nativos (García Collazo et al. 2013). El Artículo 6 de la Ley indica que las provincias deben realizar sus OTBN a través de un proceso participativo, sin especificar ni exigir los mecanismos a través de los cuales realizar dicho proceso participativo (tampoco lo hace el Decreto 91/2009). La participación genuina de los actores sociales es deseable en la medida en que propicia una distribución más equitativa de los recursos naturales del territorio (Groppo 2014). Pese a sus limitaciones, la Ley representa un hito en la legislación ambiental argentina al instalar la necesidad de planificar la distribución de actividades productivas en el territorio (García Collazo and Paruelo 2014).

Por otro lado, la Ley implementó un esquema de compensación monetaria para la conservación, lo que representa un instrumento novedoso para la gobernanza ambiental en la Argentina. El Fondo está destinado a diversos rubros que resultan clave para el funcionamiento de la Ley, como, por ejemplo, la compensación a productores por conservar o hacer uso sustentable, con o sin restricciones al cambio en el uso del suelo, el financiamiento de planes de manejo y asistencia técnica de pequeños productores y comunidades indígenas y campesinas, y el fortalecimiento de las capacidades de monitoreo y control de las autoridades de 
aplicación provincial (Artículo 35, Ley 26.331 2009). A lo largo de estos años se financiaron alrededor de 4500 planes de manejo y conservación de bosques.

El Artículo 41 de la Constitución Nacional de 1994 establece que el daño ambiental genera la obligación legal de recomposición o restauración ambiental. Luego, la Ley General del Ambiente (Ley 25.675 2002) estableció las normas que regirán los hechos o actos jurídicos, lícitos o ilícitos que, por acción u omisión, causen daño ambiental de incidencia colectiva. La Ley incluye un sistema de multas a aquellas personas físicas o jurídicas que incumplen con lo reglamentado por los OTBN provinciales. Desde la perspectiva del derecho civil, a esta multa se la considera una contravención. Sin embargo, la Ley también permitió que los daños ambientales relacionados con la pérdida o degradación del bosque sean considerados como daños jurídicos. Esto habilitó el accionar legal o judicial en los casos de alteraciones ambientales e implicando el deber de recomposición ambiental. El "Caso Karlen", en el noreste de la provincia de Salta, es un ejemplo de la aplicación de este procedimiento jurídico. Se trató de la primera vez en la Argentina en la que la Justicia provincial condenó (en octubre de 2015) a seis meses de prisión condicional por el delito de desobediencia judicial a un empresario que, al desobedecer una clausura, siguió cultivando en un predio al que antes había desmontado sin autorización. La demanda también incluye una multa que sería destinada a financiar las actividades de restauración ambiental (REDAF 2016). En este contexto, actualmente, cambió el eje de las demandas de algunos sectores, que pasaron de reclamar la existencia de una norma a exigir que los desmontes se conviertan en un delito penal. En el año 2016 una Diputada Nacional, canalizando las demandas de una ONG, presentó un proyecto de Ley que establece una pena de 2 a 10 años de prisión a quien, sin autorización, ocasione intencionalmente el desmonte, incendio, cambio de uso de suelo o la destrucción de bosques nativos (Expediente 6864-D-2016).

Desde una aproximación de legislación comparada, en los últimos años, Sudamérica experimentóavancesy reformasimportantesen cuanto a la legislación para conservar bosques nativos (FAO 2010). En particular, esto resultó clave en las ecorregiones del Gran Chaco, el Bosque Atlántico y la Selva Amazónica, en las que se pusieron en práctica diferentes aproximaciones para reducir la deforestación (Nolte et al. 2016). La Ley constituye un ejemplo novedoso, pues plantea una política de ordenamiento territorial de las áreas boscosas y un mecanismo de compensación por su conservación, instrumentos que se refuerzan entre sí de forma sinérgica.

\section{La Ley visibilizó conflictos latentes en el} territorio y fortaleció la posición de actores invisibilizados o marginados de la toma de decisiones en torno al uso del bosque nativo. Sin embargo, la implementación de los mecanismos de participación propuestos por la Ley tuvo resultados muy dispares en cuanto a la participación activa y equitativa de los actores interesados; esto pudo haber afectado negativamente su legitimidad.

Las discusiones en torno a la Ley propiciaron cierta visibilización del estado de situación de gran cantidad de pobladores de los bosques nativos, en especial, comunidades indígenas y campesinas. Estos pobladores y comunidades encontraron en la Ley un canal a través del cual manifestar sus reclamos y reafirmar su dependencia de los servicios ecosistémicos que proveen los bosques nativos. Esto permitió identificar y visibilizar conflictos históricos por la tierra y el ambiente, asignar causas y designar responsables para su recomposición y conservación. La Ley ha ofrecido un canal más a través del cual las comunidades indígenas y campesinas pueden formular y procesar las disputas por la tierra. De esta manera, permitió poner a la vista esos conflictos a través de un nuevo contexto jurídico-institucional. Esto, a su vez, habilitó la articulación de las demandas con otras experiencias de lucha y la generación de alianzas con actores estratégicos (en particular, ONG y ciertos organismos estatales) (Schmidt and Langbehn 2016).

El Artículo 6 de la Ley indica que el OTBN se debe realizar de forma participativa. La participación ciudadana en este proceso es fundamental porque, entre otras cuestiones, los usuarios del bosque aceptarán como legítimos los instrumentos de la Ley en la medida en que las decisiones de planificación resulten de procesos con altos niveles de participación (Adger 2005). La participación ciudadana en el proceso de diseño de los OTBN fue variable entre provincias. Sólo la actualización del OTBN de la provincia de Santiago del Estero tuvo participación interactiva, es decir, los diferentes actores sociales participaron activamente en el diseño del OTBN (material 
suplementario 1). Por el contrario, en la mayoría de las provincias, la participación se limitó a consultas en audiencias públicas o al suministro de información por parte de las autoridades. Incluso, para algunas provincias no está documentada la participación ciudadana.

Aunque en algunas provincias haya tenido lugar una participación activa de la ciudadanía, eso no implicó que sus opiniones fueran tomadas en cuenta en las versiones finales de los OTBN (Silvetti et al. 2013; Seghezzo et al. 2011). Esto se debió a diversas pujas de poder entre los diferentes actores sociales y a la capacidad diferencial que tuvieron para influir en la toma de decisiones (Cabrol and Cáceres 2017; Cáceres et al. 2016; Langbehn 2015; Schmidt 2013). Si bien el diseño de los OTBN habilitó la discusión entre diversos actores sociales, y prescribió la generación de ámbitos para que ocurra, no pudo pasar por alto las relaciones de poder al interior de las provincias (Seghezzo et al. 2011). En definitiva, la falta de equidad en la participación ciudadana pudo haber afectado negativamente la legitimidad de los OTBN (Seghezzo et al. 2011). Sin embargo, no existe una evaluación formal de la legitimidad de las zonificaciones finales de cada provincia. En ese sentido, queda pendiente una evaluación sistemática y de alcance comparativo sobre los procesos participativos, lo cual requiere un enfoque que tome en cuenta los alcances y limitaciones de diferentes dispositivos de participación (Blondiaux 2014).

3. En el diseño de los instrumentos de la Ley no se consideraron de manera explícita ni la estructura compleja de tenencia de la tierra subyacente ni su variabilidad entre provincias. Los cambios recientes en la implementación del Fondo comenzaron a abordar una parte de esta complejidad.

Debido a su historia de ocupación, una gran proporción de las tierras cubiertas por bosques nativos en la Región Chaqueña son el espacio vital para la reproducción social de familias campesinas y comunidades indígenas, en gran medida bajo formas precarias de tenencia de la tierra. Así, por ejemplo, en los departamentos del este de Salta donde se produjeron altas tasas de deforestación por expansión de la frontera agropecuaria, en el año 2002, 40\% de las unidades productivas eran utilizadas por pequeños productores rurales que no poseen titularidad sobre las tierras que ocupan (Reboratti 2008). Actualmente, en la misma región, sobre una superficie similar, existen conflictos en torno a la tenencia de la tierra entre productores agropecuarios, familias campesinas y comunidades indígenas (Seghezzo et al. 2017). En forma similar, en el noroeste de Santiago del Estero, 25\% de las unidades productivas son utilizadas por pequeños productores sin títulos de propiedad, bajo la presión constante, en muchos casos violenta, de grandes productores agropecuarios que quieren acaparar esas tierras para deforestar e implantar cultivos y/o pasturas (Reboratti 2008).

En este contexto, distintos autores indican que la inseguridad en la tenencia de la tierra y los conflictos derivados no tuvieron suficiente importancia en el diseño y la implementación de los instrumentos de la Ley (El OTBN y el Fondo) (Schmidt 2014; Schmidt and Langbehn 2016; Seghezzo et al. 2017). Los propios actores involucrados pusieron esto de manifiesto durante el diseño de los OTBN provinciales, al punto que, en la provincia de Salta, las comunidades indígenas y las familias campesinas, así como también los grupos ambientalistas y la comunidad académica, reclamaron suspender el proceso de zonificación hasta tanto los conflictos en torno a la tenencia de la tierra no fueran resueltos en forma satisfactoria (Seghezzo et al. 2017). Algunos gobiernos provinciales buscaron incluir los problemas de tenencia de la tierra en el diseño de los OTBN, por ejemplo, al asignar un valor de conservación medio (Categoría II) a gran parte de las áreas reclamadas por comunidades indígenas, no así a las áreas reclamadas por familias campesinas (REDAF 2012; Schmidt 2013). Incluir sus tierras en esta categoría les permitiría a estas comunidades continuar realizando su uso tradicional del bosque nativo (i.e., cría de ganado, extracción de leña y caza). Esto demuestra que los problemas de tenencia no fueron abordados en sus causas, sino que fueron incorporados a través de iniciativas orientadas a mitigarlos. Aun así, esta medida paliativa no benefició a todos los pequeños productores. Por ejemplo, en Córdoba, la clasificación de sus tierras en la Categoría II trajo aparejadas dificultades para comercializar los productos forestales que obtienen de sus actividades tradicionales (Cabrol and Cáceres 2017). En definitiva, los OTBN de las diferentes provincias encontraron dificultades para lidiar con los conflictos por la tenencia de la tierra, lo cual pone de evidencia que el instrumento no fue diseñado para abordar la compleja situación en torno a la tenencia de la tierra en la Región Chaqueña. 
En forma similar, inicialmente el Fondo no contempló la existencia de una gran cantidad de familias y comunidades que usan los bosques nativos bajo formas precarias de tenencia. En un primer momento, sólo personas que eran "titulares de derechos reales de uso, goce o disposición" podían ser beneficiarias del mismo, mediante la presentación y ejecución de planes de manejo o conservación sobre el bosque nativo (Cabrol and Cáceres 2017). En 2014, esta limitación hizo que sólo $0.7 \%$ de los beneficiarios del Fondo fueran comunidades indígenas o campesinas (material suplementario 2). En respuesta a reclamos para que la Ley cumpliera con sus objetivos de inclusión social, la resolución del Consejo Federal de Medio Ambiente (CoFeMA 277/14) creó la figura de "beneficiarios agrupados" para que aquellas familias o comunidades con un título imperfecto puedan presentar y ejecutar planes por la vía tradicional. Asimismo, esta resolución amplió el concepto de titular (y, por tanto, de potencial beneficiario del Fondo) a aquellas personas o colectivos que acrediten ser poseedores o tenedores de las tierras que utilizan. La proporción de planes ejecutados por comunidades indígenas y campesinas se duplicó en 2015 respecto a 2014 (1.3\%) (MAyDS 2016). Sin embargo, la proporción aún es baja. Este bajo porcentaje se puede atribuir a la dificultad técnica para completar los trámites administrativos por parte de ciertos actores sociales. La Ley prevé de forma explícita que una parte del 30\% del fondo que obtienen las provincias deba ser destinado a la asistencia técnica y financiera de pequeños productores y comunidades indígenas y campesinas para propender a la sustentabilidad de sus actividades (Artículo 35, Ley $\mathrm{N}^{\circ} 26.331$ 2009). Sin embargo, se desconoce en qué medida esto ocurrió. No obstante, en Córdoba, la organización social mostró ser un canal alternativo para afrontar los costos asociados a la asistencia técnica para presentar planes. Las comunidades organizadas en movimientos sociales fueron aquellas con mayor acceso al Fondo (Cabrol and Cáceres 2017)

4. Los criterios de sustentabilidad ambiental han sido abordados de forma parcial y disimil entre provincias, y los procedimientos de zonificación utilizados por cada una de éstas no han sido fundamentados ni explicitados. Como resultado ha prevalecido la dimensión económica frente a la ambiental y social, poniendo en riesgo la conectividad ecológica. Actualmente, han comenzado a proponerse mecanismos para mejorar la coherencia e integración entre las zonificaciones provinciales.

Para la realización de los OTBN, en el Artículo 6 de la Ley y su anexo, se definieron diez criterios de sustentabilidad ambiental. Siete de ellos se refieren a aspectos biológicos, dos al potencial productivo de las tierras cubiertas por bosques, y uno considera el valor atribuido al bosque por las comunidades indígenas y campesinas existentes. Estos criterios deben integrarse en un "análisis ponderado" para determinar el valor de conservación de cada sector. Sin embargo, la Ley no indica con qué indicadores deben medirse esos criterios, ni cómo deben ponderarse (Langbehn 2015). Esto llevó a que el tipo de información de base utilizada en cada caso para elaborar los OTBN y la manera de integrar esa información difieran entre provincias (Gautreau et al. 2014). Esto dio lugar a ordenamientos heterogéneos (García Collazo et al. 2015). Además, en general, las provincias no fundamentaron ni hicieron explícitos los procedimientos de zonificación utilizados, con lo cual resulta imposible reproducir el mapa realizado (Gautreau et al. 2014).

Los criterios especificados por la Ley tuvieron diferente peso para la definición de los OTBN. Por ejemplo, Griffiths y colaboradores (2014) señalaron que el criterio de potencial agrícola generalmente tuvo, implícita o explícitamente, una ponderación mayor con respecto al resto de los criterios. Análisis recientes muestran que la Categoría I fue localizada generalmente en zonas con menor presión de deforestación (i.e., áreas con bajo potencial agropecuario y menor costo de oportunidad) respecto a las áreas asignadas a la Categoría III (Nolte et al. 2017). En particular, dentro de los criterios ambientales, aquellos relacionados con el potencial de conservación de cuencas y existencia de valores biológicos sobresalientes resultaron ser los criterios empleados con mayor frecuencia por las provincias (García Collazo et al. 2013). La ponderación y la valoración diferencial de los criterios no sólo son el resultado de la adjudicación de un mayor peso relativo a la dimensión económica, sino también de la calidad deficiente o falta de información con respecto a otras dimensiones (Gautreau et al. 2014). Para varios de los criterios de sustentabilidad establecidos por la Ley, las provincias no disponían de una base cartográfica, con lo cual ni siquiera fueron utilizados. 
Todos estos elementos condujeron a que exista una gran variabilidad en cuanto a los criterios utilizados por las provincias para definir sus zonificaciones, y también en cuanto a la superficie dedicada a cada categoría de conservación. Esto determinó que los OTBN provinciales de la Región Chaqueña por lo general no incorporen a todos los bosques con alto valor de conservación dentro de las categorías que restringen la deforestación (Nolte et al. 2017). No obstante, desde el momento de aprobación de cada ley provincial de OTBN, la Autoridad Nacional de Aplicación comenzó un trabajo de consenso para ajustar aquellos aspectos de la zonificación que pudieran presentar divergencias con los objetivos de la Ley, a través de compromisos asumidos y ajustes progresivos a ser incorporados en las actualizaciones (Griffiths et al. 2014).

Si bien el decreto reglamentario de la Ley indica que el grado de detalle del OTBN debe ser de al menos de 1:250.000 (Decreto 91/2009), la mayoría de las provincias de la región no especificó la escala de los insumos que se usaron para elaborar las zonificaciones o que utilizaron de maneras diferentes a las recomendadas (Gatreau et al. 2014). A su vez, algunas provincias incorporaron la zonificación a nivel predial en sus normativas provinciales (e.g., las denominadas "recategorizaciones" en Salta). Sin embargo, al igual que para los OTBN, las metodologías utilizadas para la elaboración de la cartografía a nivel predial fueron de baja consistencia técnica e incluso en algunos casos, los procedimientos y criterios utilizados resultan confusos o se desconocen.

Por último, la zonificación realizada a escala provincial no permite incorporar la complejidad de algunos patrones que se manifiestan a mayor escala, tales como la conectividad de los bosques o la provisión y demanda de servicios ecosistémicos o, a menor escala, como los tipos forestales presentes. El mapa regional de OTBN, resultante de ensamblar el conjunto de mapas provinciales, muestra inconsistencias y deficiencias de la política de conservación a esa escala. Existen cambios abruptos entre categorías entre provincias, lo cual incrementará los problemas actuales de fragmentación y conectividad de los bosques (García Collazo et al. 2013; Piquer-Rodríguez et al. 2015). Actualmente, existe una propuesta de corredores ecológicos para la Región Chaqueña, impulsada por la Dirección Nacional de Bosques (2015), que apunta a ser un elemento complementario a los OTBN provinciales para incrementar la integración regional.

5. La Ley promueve la conservación del bosque por medio de su uso sustentable, y los OTBN provinciales asignaron una gran proporción del territorio a este fin (Categoría II). Aunque la Nación y las provincias demoraron en generar y promover alternativas viables y apropiables de uso sustentable del bosque, recientemente surgen alternativas como el Manejo de Bosques con Ganadería Integrada (MBGI) para cubrir este vacío.

Los usos del suelo permitidos en cada una de las categorías de conservación han sido motivo de intensos debates (Silvetti et al. 2013). Esto ocurrió con mayor intensidad en la Categoría II, destinada a aprovechar de forma sostenible el bosque, el turismo, la recolección y la investigación científica. A esta categoría se le asignó más del $60 \%$ de los bosques nativos en las provincias de Chaco, Santiago del Estero y Salta, tres de las provincias con mayor superficie de bosque remanente y con mayor presión por cambios en el uso del suelo de la Región Chaqueña. Para estas provincias, García Collazo y colaboradores (2013) encontraron discrepancias de peso en cuanto al nivel de transformación permitido tanto en la Categoría II como en la III. En la Categoría II, Chaco y Santiago del Estero flexibilizaron los usos permitidos dentro de estas áreas, admitiendo cambios de uso del suelo y aprovechamiento silvopastoril. Esto reduce la superficie destinada a la conservación y al manejo sostenible a un porcentaje menor a 50\% de lo asignado inicialmente (García Collazo et al. 2013). En particular, Santiago del Estero, para compatibilizar la Ley con una ley forestal provincial anterior, adjudicó "puntos verdes" dentro de la Categoría II, los cuales habilitan de forma parcial la conversión del bosque a nivel predial (Camba Sans et al. 2018). La variedad de interpretaciones de lo que la Ley define como uso sustentable, sumado a la tergiversación del término "sistemas silvopastoriles", condujo a que los bosques de la Categoría II sean en muchos casos convertidos a sistemas tipo "parque", es decir, sistemas abiertos con baja densidad de árboles dispersos. El mal uso de este término generó intensas controversias ya que bajo "sistemas silvopastoriles" se engloba a un conjunto de prácticas de manejo pastoril que producen una transformación muy variable de la estructura del bosque a través de la remoción selectiva de árboles y arbustos. En definitiva, los OTBN provinciales encontraron 
dificultades o deficiencias para instrumentar efectivamente el uso sustentable del bosque establecido por la Ley.

En respuesta a la situación descripta anteriormente, se firmó un convenio entre la ex Secretaría de Ambiente y Desarrollo Sustentable de la Nación y el ex Ministerio de Agricultura, Ganadería, Pesca y Alimentos de la Nación (Convenio 32/2015). El objetivo de este convenio fue establecer pautas específicas que permitieran compatibilizar el manejo ganadero y forestal de los sistemas silvopastoriles con la conservación de los bosques nativos en aquellas categorías que permiten alteraciones, totales o parciales, del bosque (categorías II y III). De esta manera, la Ley propició un contexto de oportunidad para ciertos proyectos de investigación y extensión orientados a generar nuevas alternativas de manejo, como por ejemplo el Manejo de Bosques con Ganadería Integrada (MBGI). Este sistema de manejo define pautas generales de manejo productivo y ecológico que no pueden aplicarse de manera idéntica en todas las regiones, sino que tienen que ser adaptadas a cada ecosistema boscoso en particular. En caso de autorizarse, su correcta aplicación demandará la colaboración entre productores, instituciones gubernamentales, ONG y organismos de ciencia y técnica. Actualmente, parte de estos esfuerzos interinstitucionales se encuentran en marcha (e.g., Aprile et al. 2016; INTA 2015).

6. La Ley es la única ley de presupuestos mínimos que se encuentra financiada. Sin embargo, el presupuesto anual asignado para su implementación se ha reducido progresivamente en relación a lo estipulado por la Ley. A su vez, los montos del Fondo de compensación no proveen incentivos económicos para reducir la deforestación.

De las numerosas leyes de presupuestos mínimos que han sido sancionadas desde la reforma constitucional de 1994, la Ley es la única que actualmente tiene financiamiento. Sin embargo, las partidas asignadas al Fondo desde el año 2010 hasta la actualidad fueron menores a $0.3 \%$ del presupuesto nacional, por lo cual se incumplió con lo estipulado en el Artículo 31 de la Ley (material suplementario3, Tablas 1 y 2). Entre el 2010-2015, la proporción asignada del monto establecido por Ley fue disminuyendo, lo cual se vio acentuado por el ingreso incremental de la totalidad de las provincias al Fondo. En el año 2010 se asignó $36 \%$ de lo establecido por la Ley, mientras que en 2016 este porcentaje disminuyó a 5\%. En 2017, el monto asignado duplicó los valores de los años anteriores y el proyecto de ley de presupuesto para el año 2018, recientemente presentado al Congreso, asigna al Fondo 556.5 millones de pesos (igual que en 2017), aunque esta cifra representa sólo $15 \%$ de lo contemplado por la Ley.

A nivel provincial, se presentaron dificultades en la ejecución de los fondos otorgados debido principalmente a dificultades para su rendición. Como resultado, la subejecución de lo presupuestado fue alrededor de $30 \%$ desde 2010, y las provincias no pudieron rendir en tiempo y forma las partidas asignadas. Sin embargo, los procedimientos vinculados a la rendición de fondos tanto por parte de las Autoridades Locales de Aplicación como de los beneficiarios particulares han sido actualizados. Se incorporó a la certificación de obras (presentada como declaración jurada por parte de las Autoridades Locales de Aplicación) como instrumento de verificación de la realización de los planes de conservación y de manejo y el requisito de presentar un informe anual que describa los fondos utilizados y las metas alcanzadas (Artículo 11 y anexo I Ley 26.331; Resolución SAyDS 826/ 14 2014). Mediante este mecanismo se busca tanto agilizar el proceso de rendición como también incrementar el nivel de fiscalización de los planes aprobados.

La definición del monto del pago a tenedores de bosque por la ejecución de planes no considera el costo de oportunidad en el que incurre el titular de las tierras ni la situación económica que atraviesan muchos actores sociales. En la práctica, $70 \%$ del Fondo es distribuido entre los tenedores de bosque de acuerdo a los gastos en materiales para la ejecución de cada plan, es decir, son compensados "en especie". De esta manera, aún para aquellos que pudieron acceder al Fondo, este instrumento no les brindó un tratamiento equitativo ya que, por ejemplo, un tenedor debosque con una situación económica desfavorable no puede utilizar los beneficios del fondo para gastos de subsistencia. A su vez, un plan de conservación ejecutado en áreas con alto potencial agrícola (mayor probabilidad de conversión) o áreas críticas para la provisión de servicios ecosistémicos (e.g., zona riparia o cabecera de cuenca), posiblemente recibió la misma compensación por hectárea que un plan de conservación ejecutado en cualquier otra zona. Finalmente, si bien está previsto que la Categoría I reciba una mayor compensación 
por hectárea conservada que las categorías II o III, esto no se traduce en un mayor incentivo para conservar. Esto se debe a que las categorías menos restrictivas admiten la ejecución de actividades productivas que resultan en un beneficio económico mayor para estas zonas y, por lo tanto, se reduce la efectividad del incentivo para conservar.

7. Para la implementación de la Ley se lograron establecer mecanismos de coordinación entre autoridades nacionales y provinciales que permitieron viabilizar el esquema de los "presupuestos mínimos" establecido en la Constitución Nacional. Noobstante, seevidenciaron diferentes niveles de implementación de la Ley en cada provincia, lo cual puede comprometer el cumplimiento de sus objetivos.

La Constitución Nacional de 1994 (Artículo 41) creó un esquema de competencias concurrentes entre Nación y las provincias en materia ambiental: mientras que la Nación tiene la facultad de dictar normas de presupuestos mínimos de protección, corresponde a las provincias dictar las normas necesarias para "complementarlas", y son ellas las que aplican en el terreno de las políticas ambientales. A la vez, las provincias detentan el dominio originario de los recursos naturales (Artículo 124). Este esquema hace indispensable cierta articulación entre las autoridades nacionales y provinciales para la implementación de normas ambientales de alcance nacional. Sin embargo, antes de la sanción e implementación de la Ley, esta posibilidad se encontraba bloqueada. Las provincias, a través del CoFeMA, habían impugnado la validez de todas las leyes de presupuestos mínimos sancionadas entre 2002 y 2004, incluyendo la Ley General del Ambiente (Ley 25.675 2002). El argumento detrás de esta decisión era que este tipo de leyes avanzaban sobre competencias reservadas a las provincias (Resolución CoFeMA 80). Sostenían, además, que las normas de presupuestos mínimos debían ser únicamente leyes sancionadas por el Congreso y que su reglamentación no le correspondía al Poder Ejecutivo Nacional (Resolución CoFeMA 92).

A partir de la sanción de la Ley se comenzó a generar un mecanismo de coordinación entre nación y provincias. La redacción del decreto reglamentario (Decreto 91/2009) fue convenida entre el Poder Ejecutivo Nacional y el CoFeMA; desde allí, este órgano se posicionó como un ámbito de negociación entre la Autoridad Nacional de Aplicación y sus equivalentes provinciales. A partir de este decreto se pudieron acordar diversos aspectos referidos a la aplicación de la Ley, tales como la distribución del Fondo, los contenidos mínimos comunes que las provincias exigen para los planes de manejo y conservación de bosques (Resoluciones CoFeMA 229 y 277), las pautas para realizar la cartografía de los bosques nativos que se aplicarán en los OTBN (Resolución CoFeMA 230) y una serie de pautas metodológicas comunes para la actualización de los OTBN para lograr una mayor homogeneidad entre provincias (Resolución CoFeMA 236). Más allá de las soluciones concretas adoptadas en estos acuerdos, la modalidad de coordinación ensayada permite viabilizar el esquema de los presupuestos mínimos a través de un "federalismo ambiental negociado" (Langbehn 2017).

A pesar de todos los esfuerzos de coordinación mencionados, la mayor parte de estas resoluciones se tomaron después de que muchas provincias tuvieran aprobados sus OTBN. Por lo tanto, su contribución a la homogeneidad y coherencia de los OTBN fue limitada. En efecto, mientras que algunas de las provincias de la Región Chaqueña concluyeron sus OTBN entre uno y dos años después de sancionada la Ley (Salta, Santiago del Estero y Chaco), otras demoraron entre tres y cinco años. Hasta la actualidad, sólo Santiago del Estero y San Juan completaron el proceso de actualización del OTBN (Material Suplementario 1).

La falta de coordinación inicial y la limitada influencia que tiene la Autoridad Nacional de Aplicación para determinar políticas de uso del suelo llevaron a que cada provincia tuviera un amplio margen de acción para definir el OTBN (Nolte et al. 2017). Por otro lado, los acuerdos políticos entre los distintos niveles de gobierno nacional y provincial no han resultado lo suficientemente fuertes como para implementar coordinadamente la Ley tomando posiciones únicas frente a todos los sectores y regiones. Esto condujo a diversas falencias. En primer lugar, las provincias aplicaron de manera muy diversa los criterios de sustentabilidad ambiental definidos por la Ley, asignando por lo tanto distintas categorías de conservación a bosques con características similares ubicados a ambos lados de las fronteras provinciales (García Collazo et al. 2013). En segundo lugar, los OTBN provinciales en algunos casos redujeron las restricciones al uso definidas 
en las categorías de conservación de la Ley, ya sea a través de las propias leyes provinciales o de actos administrativos posteriores. Por ejemplo, en Salta se permitió cambiar de categoría ("recategorizaciones") áreas con mayor restricción al uso (categorías I y II) hacia categorías con menor restricción (Categoría III). En Santiago del Estero se implementaron los puntos verdes descriptos anteriormente, y en Chaco se permitió deforestar una proporción de los bosques presentes en la Categoría II (Camba Sans et al. 2018; García Collazo et al. 2013; Nolte et al. 2017). En Formosa, el OTBN determina conservar una proporción variable de los bosques incluso en la Categoría III y por ello, la provincia asignó aproximadamente $70 \%$ de su superficie forestal a esta categoría (García Collazo et al. 2013).

8. A nivel nacional y provincial, los organismos encargados de la gestión de los bosques nativos aumentaron sus capacidades de control y fiscalización. Sin embargo, estas capacidades siguen siendo insuficientes, lo que dificulta tanto reducir las tasas de deforestación ilegal como mejorar el estado de conservación de los bosques nativos.

En términos institucionales, los organismos provinciales y nacionales encargados del control y la gestión de los bosques nativos se fortalecieron con la incorporación de personal especializado y la estructuración de sistemas de información, además de contar con acceso a otros recursos materiales (e.g., vehículos). Actualmente, estos cambios ofrecen posibilidades para la gestión y control de los bosques nativos superiores a los existentes previamente a la sanción de la Ley. Por ejemplo, algunas provincias no contaban con dependencias encargadas del manejo de los bosques nativos, y la Ley les permitió dar un marco para establecer los organismos de control a nivel provincial. Si bien la información sobre los mecanismos y esfuerzos que cada provincia ha empleado al control del cumplimiento de la Ley es escasa, indicadores indirectos como la superficie deforestada en Categoría I y II, sugieren que estos controles resultaron insuficientes (Camba Sans et al. 2018; Volante and Seghezzo 2018). Por otro lado, dada la vasta extensión de los bosques nativos, un control in situ exhaustivo no es una alternativa viable. En este contexto, además del monitoreo de la deforestación realizado por la Dirección de Nacional de Bosques, en los últimos años surgieron otras instituciones que realizan monitoreos en base a información satelital (e.g., monitoreodesmonte.com.ar).
Respecto a la penalización por incumplimiento, que también se encuentra a cargo de las provincias, la información disponible está publicada en el Registro Nacional de Infractores. Actualmente, este registro sólo cuenta con los aportes de cinco provincias y el número de sanciones que contiene es mínimo respecto al área deforestada en categorías no permitidas. A su vez, la variabilidad en los montos y en las sanciones aplicadas permite cuestionar la eficacia que tienen las mismas para evitar mayores infracciones a la Ley, sobre todo teniendo en cuenta que los beneficios económicos derivados de la producción agropecuaria pueden superar ampliamente los costos que suponen las sanciones. La eficacia de las actividades de control y fiscalización depende de la capacidad de identificar el desmonte ilegal y de sancionar al infractor con una pena que suponga un monto superior a los beneficios que deriva de sus actividades productivas. Esto último no sucede, ya que los desmontes sin autorización, o en zonas no permitidas, en el ámbito del derecho penal administrativo, son simplemente contravenciones para las que se establecen multas que imponen costos cuyo monto no constituye un desincentivo a la deforestación ilegal. A su vez, además de los mecanismos de contralor y de los esfuerzos de fiscalización, es importante el papel que cumple la corrupción por parte de funcionarios provinciales. Por ejemplo, en el caso de la provincia de Córdoba, el mal desempeño de funcionarios encargados de autorizar y controlar la deforestación y de sancionar a aquellos que lo hacen ilegalmente, derivaron en la renuncia de funcionarios de alta jerarquía (Cabrol and Cáceres 2017).

La Autoridad Nacional de Aplicación desarrolló un sistema de fiscalización de planes de manejo sostenible financiados por la Ley. Las comisiones de verificación y control de planes tienen el propósito de verificar en el terreno las condiciones indicadas del bosque y la ejecución de las acciones propuestas. El total de planes fiscalizados a la fecha en todo el país es 245, entre proyectos de formulación, planes de manejo y planes de conservación. El resultado de este proceso de fiscalización se traduce en informes de observaciones y propuestas de mejora de los planes para las Autoridades Locales de Aplicación ya que la facultad para el control y toma de decisiones reside finalmente en las provincias. En muchas ocasiones, esto resulta problemático porque los organismos locales responsables de fiscalizar el cumplimiento de la Ley están 
subordinados, o son de menor jerarquía, que los organismos promotores de la expansión de la frontera agropecuaria.

9. En la Región Chaqueña, las tasas de deforestación se redujeron durante el período de diseño e implementación de la Ley. Sin embargo, esta disminución no puede ser atribuida únicamente a factores vinculados a la aplicación de esta norma.

En coincidencia con la implementación de la Ley, las provincias con mayores tasas de deforestación del Chaco argentino (Santiago del Estero, Salta y Chaco) presentaron una disminución de sus tasas de deforestación (Camba Sans et al. 2018; Nolte et al. 2017; Volante and Seghezzo 2018). Sin embargo, no existe consenso respecto a si este descenso se debe a la aplicación de la Ley o a otros factores nacionales e internacionales. En primer lugar, Volante y Seghezzo (2018) indican que la disminución en las tasas de deforestación comenzó antes de la entrada en vigencia de la Ley. A su vez, estos autores y Camba Sans et al. (2018) analizaron la efectividad de la normativa en Santiago del Estero, y sus resultados sugieren que la Ley no tuvo el efecto deseado pues hubo una pérdida significativa de bosques en sitios donde la conversión de bosques está prohibida, es decir, categorías I y II. Además, no todas las provincias del Chaco argentino presentaron disminuciones en sus tasas de deforestación luego de la implementación de la Ley. En Formosa, la cuarta provincia con mayores niveles de deforestación, ésta se incrementó luego de la entrada en vigencia de la Ley (Volante and Seghezzo 2018).

Durante la primera década del siglo XXI, la deforestación en el Chaco argentino estuvo altamente asociada al precio de la soja (Gasparri et al. 2013). Sin embargo, luego de alcanzar un máximo en 2012, el precio disminuyó y actualmente es aproximadamente la mitad (bit.ly/2JPgIlo). El maíz, otro cultivo ampliamente extendido en la región chaqueña asociado con la ganadería, presentó el mismo patrón quela soja. Estas disminuciones podrían explicar de forma parcial la reducción en la tasa de deforestación que se observó en los últimos años, aunque en la actualidad, este proceso comenzó a ser impulsado en mayor medida por la ganadería (i.e., conversión de bosques a pasturas) (Fehlenberg et al. 2017; Gasparri et al. 2013). Por lo tanto, en un escenario de aumento en el precio de commodities, las tendencias en las tasas de deforestación observadas en los últimos años podrían revertirse y aumentar. Sin embargo, es probable que no alcancen los niveles observados en los años previos a la sanción de la Ley de Bosques debido a que: a) la mayor parte de las tierras con aptitud para realizar agricultura de secano ya fueron transformadas y están en uso (Adámoli et al. 2011), b) el avance de la frontera agropecuaria muestra cierta tendencia a desplazarse hacia áreas o países con menores restricciones a la deforestación (de Waroux et al. 2016) y c) la movilización social en contra de la pérdida de bosques nativos (empoderada por la Ley de Bosques) y las campañas y denuncias de ONG representan un medio de control social de la deforestación (Aguiar et al. 2016).

10. Existen vacíos de información que impiden evaluar el desempeño de la Ley más allá de la superficie deforestada o de la ejecución de fondos. La evaluación de la equidad, legitimidad y flexibilidad de la Ley constituye un desafío.

En torno a la sanción y la aplicación de la normativa se ha dinamizado la generación de conocimiento sobre los bosques nativos y su uso, sobre el proceso de deforestación y sobre sistemas productivos de bajo impacto ambiental, que permiten hoy enfrentar con mejores herramientas los desafíos que implica su conservación. Del mismo modo, desde la Dirección de Bosques de Nación se incentivó el sector de ciencia y técnica para orientarlo hacia la generación de información básica y aplicada sobre diversos aspectos de los bosques nativos que se vinculan directamente con la implementación de la Ley. Un ejemplo de ello son los Proyectos de Investigación Científica y Tecnológica Orientados al manejo sustentable de los bosques nativos (PICTOs) que surgen de la acción coordinada entre la Agencia Nacional de Promoción Científica y Tecnológica (ANPCyT) y la ex Secretaría de Ambiente y Desarrollo Sustentable.

Los esfuerzos de monitoreo y evaluación de la Ley estuvieron enfocados en cuantificar y localizar los cambios en la superficie de bosque nativo a escala regional. Si bien la protección de los bosques nativos remanentes es uno de los principales objetivos de la Ley, evaluar el desempeño de un instrumento de política pública sólo en función de la consecución de un objetivo a una única escala espacial no es suficiente al menos por dos razones (Adger et al. 2005). Primero, la consecución de un objetivo a una determinada escala puede generar externalidades negativas y desigualmente distribuidas a otras escalas espaciales o 
temporales. Por ejemplo, una acción que desacelera la tasa de deforestación a escala regional puede estar imponiendo mayores restricciones de uso de la tierra a escala predial a productores pequeños y medianos con respecto a los productores grandes. Segundo, la consecución de un objetivo por un determinado actor social puede generar un desplazamientodelasexternalidadesnegativas a otros actores sociales o áreas geográficas. Por ejemplo, una acción que desacelera la tasa de deforestación en una provincia o país puede estar aumentando la deforestación en otra provincia o país por migración de grandes empresas agropecuarias en busca de áreas con menores restricciones de uso de la tierra, en un fenómeno llamado desplazamiento trans-fronterizo del cambio en el uso de la tierra (de Waroux et al. 2016). Para superar estas limitaciones, los principios generales de la evaluación de políticas sugieren ampliar el abanico de indicadores de desempeño utilizados, de manera de orientar decisiones que sean efectivas, pero además equitativas, legítimas y flexibles (Adger et al. 2005).

La efectividad del Fondo en términos de conservación de los servicios ecosistémicos brindados por el bosque nativo y el fortalecimiento institucional no ha sido evaluada aún, mientras que la efectividad de los OTBN fue evaluada únicamente en relación con la superficie de bosques a escala regional y provincial (UMSEF 2016). Una de las pocas fuentes que aporta información sobre sus efectos distributivos es aquella sobre la asignación de los recursos del Fondo entre distintos tipos de titulares, en sentido amplio, de la tierra. Los análisis existentes coinciden en que la Ley está desnudando los conflictos existentes por la tenencia de la tierra y la vulnerabilidad de las comunidades indígenas y campesinas, visibilizando esta situación y generando nuevos ámbitos de discusión; sin embargo, en la mayoría de los casos no logró reducir las desigualdades históricas y preexistentes a la Ley (Cabrol and Cáceres 2017; Langbehn 2016; Seghezzo et al. 2017). Si bien los instrumentos jurídicos no resuelven por sí diferentes problemas estructurales y la asimetría en el acceso a los recursos naturales (Azuela 2006; Merlinsky 2013), estas condiciones preexistentes pueden afectar negativamente la legitimidad de la Ley. Sin embargo, no existe información sistemática que indique el grado de aceptación que los actores involucrados tienen respecto de las normas establecidas por la Ley.
Las señales que indican un desempeño deficiente de la Ley en términos de efectividad, equidad y legitimidad podrían ser revertidas en la medida en que sus instrumentos tengan la flexibilidad necesaria para que, luego de una evaluación de las metas intermedias y garantizando niveles de participación efectiva, sea posible reformular los aspectos que presentaron resultados regresivos. La Ley establece quelosOTBN deben actualizarse cada cinco años mediante un proceso participativo, con el objetivo de otorgar flexibilidad a este instrumento y contribuir a la progresividad ambiental. Dado que la mayoría de las provincias tenía aprobado su OTBN inicial a fin de 2011, habría correspondido que la actualización se realizara en el año 2016.

\section{Propuestas para mejorar el DESEMPEÑO DE LA LEY DESDE LA INTERFAZ INVESTIGACIÓN-GESTIÓN}

Las diez observaciones precedentes ponen de manifiesto oportunidades para reformular la Ley al consolidar sus fortalezas y minimizar sus debilidades, a partir de, entre otras, la identificación de los vacíos de información que existen en cuanto a su diseñoe implementación. Para mejorar su desempeño es necesario el accionar conjunto y la vinculación entre los distintos sectores de la sociedad, de manera de promover sinergias entre los componentes técnico, político y administrativo que conforman las políticas ambientales (Paruelo 2016). El sector político tiene a su cargo la gestión de las tierras boscosas y la efectividad de dicha gestión requiere, como se dijo, una fuerte coordinación y compromiso del Estado en sus niveles nacional y provincial, lo cual es una condición necesaria para una fuerte integración horizontal del Estado con la comunidad científica y la sociedad civil. Cada vez es mayor el reconocimiento de que la vinculación entre el sector político y la comunidad científica resulta clave para diseñar estrategias de gestión efectivas, eficientes, equitativas y legítimas (Gautreau et al. 2014). Este espacio de vinculación se conoce como la interfaz investigación-gestión (Roux et al. 2006), y puede jugar un rol fundamental en la generación de información y conocimiento aplicable al (re)diseño y ajuste de los instrumentos de la norma. La Ley fortaleció la vinculación entre la investigación y la gestión de los bosques nativos, y es en ese contexto que resulta oportuno identificar líneas de acción para ser abordadas desde esta interfaz, las 
cuales permitan conformar una agenda común respecto a sus direcciones futuras. La responsabilidad de llevar adelante estas acciones recae, en mayor o menor medida, en uno $\mathrm{u}$ otro sector de acuerdo a sus capacidades y mandatos; pero, en todos los casos, el flujo bidireccional de información y conocimiento en la interfaz resulta clave para el diseño y la ejecución de estas acciones (Roux et al. 2006). A continuación, resumimos en una lista no exhaustiva, diez propuestas concisas que refieren a las líneas de acción que a nuestro entender contribuirían significativamente a cubrir los vacíos de investigación y corregir algunas de las limitaciones identificadas en las observaciones precedentes:

(1) Incluir en la evaluación del desempeño de la Ley aspectos relacionados con su legitimidad e impactos sobre la equidad social. Ampliar la evaluación de su efectividad mediante el relevamiento de indicadores del estado de conservación del bosque nativo, de su capacidad de proveer servicios ecosistémicos y de la calidad de vida de sus habitantes desagregando por tipo de actor social. En este sentido, se podría implementar un sistema de monitoreo satelital de los bosques nativos remanentes. Esto sería útil para caracterizar los cambios asociados a la implementación de los planes de manejo y conservación y también para determinar áreas prioritarias para establecer este tipo de planes.

(2) Mejorar los mecanismos de participación ya sea para procesos de difusión, actualización o consulta y hacer una evaluación de los mismos. Para ello, es necesario identificar a todos los actores sociales relevantes y conocer de manera más sistemática y rigurosa sus opiniones, perspectivas y propuestas con el objetivo de evitar que la toma de decisiones se concentre en los actores con más poder (político y/o económico) y mejor acceso a los medios de difusión masiva. Instrumentar mecanismos (e.g., técnicas multicriterio) que permitan incorporar las visiones y valores de diversos actores sociales en la ponderación de los criterios para realizar las actualizaciones de los OTBN.

(3) Evaluar en forma sistemática la ocurrencia de conflictos en torno a la tenencia y uso de la tierra, y respecto del acceso a los servicios ecosistémicos provistos por el bosque nativo. Incorporar esta información en la revisión y el rediseño de los instrumentos de la Ley, de manera de priorizar la resolución de los conflictos y evitar que se intensifiquen a causa de las regulaciones e incentivos de la Ley.
(4) Revisar la selección y la ponderación de los criterios de sustentabilidad ambiental para la zonificación de los bosques nativos para que la asignación de las categorías de conservación contribuya a alcanzar de forma progresiva las metas y objetivos de la Ley en el marco de la actualización de los OTBN. Generar bases de datos con información espacialmente explícita que faciliten la aplicación de los criterios de sustentabilidad ambiental. Promover la protocolización del proceso de zonificación en el marco de las actualizaciones para poder cuantificar la importancia relativa que se le otorga a cada uno de los criterios.

(5) Diseñar criterios y metodologías para caracterizar el valor de conservación de los bosques a nivel de paisaje, e incorporar dichos valores como criterios para la zonificación a escala regional.

(6) Intensificar los esfuerzos para evaluar la sustentabilidad de los sistemas de uso del suelo permitidos en cada categoría de conservación, en especial aquellos permitidos en la Categoría II, como los sistemas silvopastoriles y el Manejo de Bosque con Ganadería Integrada.

(7) Garantizar la adicionalidad en los OTBN y el Fondo, de manera que se les asigne la mayor categoría de conservación a aquellas áreas cubiertas por bosques nativos cuya biodiversidad y provisión de servicios ecosistémicos se encuentre bajo la mayor amenaza de conversión a otros usos. Para esto es posible combinar fuentes de información disponible acerca de la probabilidad actual y futura de conversión de los bosques a usos agropecuarios (e.g., Gasparri et al. 2015; Volante et al. 2016) y el nivel de provisión de servicios ecosistémicos (Paruelo et al. 2016).

(8) Mejorar el control y la fiscalización de la deforestación ilegal mediante el diseño y la implementación de un mecanismo de alerta temprana de desmontes. Este mecanismo puede estar basado en información provista por sensores remotos, como el desarrollado en Brasil por INPE (DETER-B) (Diniz et al. 2015) o por un sistema organizado de denuncias a modo de "alerta ciudadana temprana" (e.g., www.forestwatchers.net; Arcanjo et al. 2016). Asociar la alerta temprana con un sistema de control efectivo en terreno, capaz de fiscalizar las contravenciones o delitos forestales. Al igual que en la propuesta anterior, los esfuerzos de control y fiscalización deberían enfocarse en áreas con mayor probabilidad de conversión y de provisión de servicios ecosistémicos. 
(9) Diseñar e implementar planes, programas y proyectos de comunicación social sobre el valor de los bosques nativos para aumentar el conocimiento y la conciencia ambiental en la población, especialmente la urbana.

(10) Diseñar estrategias de gestión transfronteriza del territorio entre países vecinos que comparten eco-regiones (e.g., Argentina, Paraguay y Bolivia para el Gran Chaco sudamericano), las cuales incluyan instrumentos de regulación o incentivos dirigidos a frenar el desplazamiento de la deforestación a través de las fronteras (Garrett et al. 2017).

Estas propuestas apuntan a desarrollar nuevas herramientas conceptuales y metodológicas que contribuyan a comprender mejor los procesos socioambientales que influyen sobre la implementación de la Ley. En cierta medida, reflejan los vacíos de conocimiento o las limitaciones de información que actualmente existen, y sugieren nuevas líneas de investigación y de acción. Sin embargo, no pretenden presentar una visión simplificada de las complejidades de la interfaz investigación-gestión ni tampoco minimizar las dificultades que tendrá que enfrentar la implementación de la Ley para lograr materializar las diferentes dimensiones que proponen Adger y colaboradores (e.g., equidad, flexibilidad, legitimidad, 2005). Las disputas de valores e intereses entre actores sociales que emergieron a partir de la promulgación de las leyes provinciales pusieron de manifiesto las asimetrías de poder y de acceso a la información, y las visiones contrapuestas de desarrollo que existen en los territorios (Cabrol and Cáceres 2017; Seghezzo et al. 2011).

A pesar de la complejidad del contexto, el conocimiento científico-técnico está en condiciones de realizar aportes y contribuir para que la Ley cumpla más ajustadamente los objetivos por los que fue creada. Pero, al mismo tiempo, es necesario reconocer que el conocimiento científico-técnico per se presenta limitaciones para influir de un modo efectivo a la esfera política. No se trata sólo de que los científicos hagan llegar la información relevante, en el formato adecuado, a las personas indicadas y en el momento correcto. La arena política es un campo muy disputado en el que confluyen múltiples actores que defienden valores, intereses y subjetividades diferentes, y en el cual las relaciones de poder juegan un rol central (Cáceres et al. 2016). En este tipo de procesos, es clave que los científicos expliciten su posición ideológica y el rol que asumen, que puede ir desde generar o "arbitrar" la información hasta promover alternativas o ser abogados de una causa particular (Paruelo 2016). La capacidad de los científicos de interactuar en forma honesta con los actores del proceso y participar activamente en la disputa de valores e intereses contribuirá a que la interfaz entre la investigación y la gestión sea capaz de transformar positivamente la implementación de la Ley.

Agradecimientos. Este trabajo fue financiado por el proyecto PICTo 2014-0046 "Bosques nativos, servicios ecosistémicos y bienestar humano en el Norte del Chaco Seco argentino: un abordaje interdisciplinar de la vulnerabilidad y los conflictos socioecológicos" (IR: Matías Mastrangelo) y por el Inter American Insitute (IAI) for Global Change Research, que es financiado por la US National Science Foundation (Grant GEO-1128040), a través del proyecto CRN3095 (Bridging Ecosystem Services and Territorial Planning (BEST-P): A southern South American intiative). Agradecemos a Ignacio Gasparri, Eduardo Manghi y Silvia Matteucci y a dos revisores anónimos cuyos comentarios contribuyeron a mejorar este artículo.

\section{REFERENCIAS}

Adámoli, J., R. Ginzburg, and S. Torrella. 2011. Escenarios productivos y ambientales del Chaco Argentino: $1977-2010$. Buenos Aires: FCEN-UBA y Fundación Producir Conservando.

Adger, W. N., N. W. Arnell, and E. L. Tompkins. 2005. Successful adaptation to climate change across scales. Global Environmental Change 15(2):77-86.

Aguiar, S., M. Texeira, J. M. Paruelo, and M. E. Román. 2016. Conflictos por la tenencia de la tierra en la provincia de Santiago del Estero y su relación con los cambios en el uso de la tierra. Pp. 199-225 en Marcela E. Román y M. C. González (eds.). Transformaciones agrarias argentinas durante las últimas décadas. Una visión desde Santiago del Estero y Buenos Aires. Editorial Facultad de Agronomía, Buenos Aires, Argentina.

Amdan, M. L., R. Aragón, E. G. Jobbágy, J. N. Volante, and J. M. Paruelo. 2013. Onset of deep drainage and salt mobilization following forest clearing and cultivation in the Chaco plains (Argentina). Water Resources Research, 49(10):6601-6612.

Aprile, G., M. E. Periago, and F. O. Miñarro. 2016. La fauna y los silvopastoriles del Chaco. Boletín técnico. Fundación 
Vida Silvestre Argentina. Buenos Aires, Argentina.

Arcanjo, J. S., E. F. Luz, Á. Fazenda, and F. M. Ramos. 2016. Methods for evaluating volunteers' contributions in a deforestation detection citizen science project. Future Generation Computer Systems 56:550-557.

Azuela, A. 2006. Visionarios y pragmáticos: una aproximación sociológica al Derecho ambiental. Editorial Fontanamara, México D.F., México.

Balvanera, P., M. Uriarte, L. Almeida-Leñero, A. Altesor, F. DeClerck, T Gardner, et al. 2012. Ecosystem services research in Latin America: The state of the art. Ecosystem Services 2:56-70.

Blondiaux, L. 2014. El nuevo espíritu de la democracia. Actualidad de la democracia participativa. Editorial Prometeo, Buenos Aires, Argentina.

Boyce, J. K. 2002. The political economy of the environment. Edward Elgar Publishing, Londres, Inglaterra.

Barbetta, P. 2005. El movimiento campesino en Santiago del Estero: luchas y sentidos en torno a la problemática de la tierra. Pp. 443-448 en N. Giarraca y M. Teubal. El campo argentino en la encrucijada. Estrategias y resistencias sociales, ecos en la ciudad. Alianza Editorial, Buenos Aires, Argentina.

Cabrol, D. A., and D. M. Cáceres. 2017. Las disputas por los bienes comunes y su impacto en la apropiación de servicios ecosistémicos: La Ley de Protección de Bosques Nativos, en la Provincia de Córdoba, Argentina. Ecología Austral 27(1):134-145.

Cáceres, D. M. 2015. Accumulation by dispossession and socio-environmental conflicts caused by the expansion of agribusiness in Argentina. Journal of Agrarian Change 15(1):116-147.

Cáceres, D. M., F. Silvetti, and S. Díaz. 2016. The rocky path from policy-relevant science to policy implementation - a case study from the South American Chaco. Current Opinion in Environmental Sustainability 19:57-66.

Camba Sans, G., S. Aguiar, V. Vallejos, and J. M. Paruelo. 2018. Assessing the effectiveness of a land zoning policy in the Dry Chaco. The Case of Santiago del Estero, Argentina. Land Use Policy 70(2018):313-321.

Consejo Federal de Medio Ambiente (CoFeMA). 2012. Resolución 236/2012. Aprobación del documento "Pautas metodológicas para las actualizaciones de los Ordenamientos Territoriales de los Bosques Nativos". Argentina

Consejo Federal de Medio Ambiente (CoFeMA). 2014. Resolución N²77/2014. Aprobación del Reglamento de Procedimientos Generales Ley № 26.331. Argentina

Costantino, A. 2016. ¿Quiénes son y para qué? el proceso de extranjerización de la tierra en argentina a partir de 2002. Ambiente y Sostenibilidad 5(1):43-56.

De Dios, R. 2006. Expansión agrícola y desarrollo local en Santiago del Estero. VII Congreso Latinoamericano de Sociología Rural. Quito, Ecuador.

Decreto 91/2009. 2009. Protección Ambiental de los Bosques Nativos. Apruébase la Reglamentación de la Ley Nº 26.331. [Consulta: 30 de abril de 2016]. URL: bit.ly/2MIltes.

de Waroux, Y. L. P., R. D. Garrett, R. Heilmayr, and E. F. Lambin. 2016. Land-use policies and corporate investments in agriculture in the Gran Chaco and Chiquitano. Proceedings of the National Academy of Sciences 113(15):40214026.

Diniz, C. G., A. A. de Almeida Souza, D. C. Santos, M. C. Dias, N. N. da Luz, D. R. V. de Moraes, et al. 2015. DETER-B: The new Amazon near real-time deforestation detection system. IEEE Journal of Selected Topics in Applied Earth Observations and Remote Sensing 8(7):3619-3628.

Dirección de Bosques. 2015. Corredores ecológicos para el Chaco Argentino. Definición y pautas metodológicas para su definición. Subsecretaría de Planificación y Política Ambiental. Secretaría de Ambiente y Desarrollo Sustentable. Argentina

Domínguez, D., P. Lapegna, and P. Sabatino. 2006. Un futuro presente: las luchas territoriales. Nómadas 24:240-246.

Engel, S., S. Pagiola, and S. Wunder. 2008. Designing payments for environmental services in theory and practice: An overview of the issues. Ecological Economics 65(4):663-674.

Expediente 6864-D-2016. 2016. Proyecto de Ley: Régimen penal de protección al bosque nativo. En línea. [Consulta: 25 de agostos de 2017]. URL: bit.ly/2MG279L.

Fairhead, J., M. Leach, and I. Scoones. 2012. Green Grabbing: a new appropriation of nature? Journal of Peasant Studies 39(2):237-261.

FAO. 2010. Leyes forestales en América del Sur. Documento de trabajo. Santiago, Chile. URL: bit.ly/2lkjQqL.

Fehlenberg, V., M. Baumann, N. I. Gasparri, M. Piquer-Rodríguez, G. Gavier-Pizarro, and T. Kuemmerle. 2017. The role of soybean production as an underlying driver of deforestation in the South American Chaco. Global Environmental Change 45:24-34.

García Collazo, M. A., A. Panizza, and J. M. Paruelo. 2013. Ordenamiento Territorial de Bosques Nativos: Resultados de la Zonificación realizada por provincias del Norte argentino. Ecología Austral 23(2):97-107.

García Collazo, M. A., and J. M. Paruelo. 2014. Ordenamiento territorial de bosques nativos en Argentina: Resultados de la zonificación en Argentina. En: Ordenamiento Territorial: Conceptos, Metodologías y Experiencia. En: J. M. Paruelo, E. G. Jobbágy, P. Laterra, H. Diéguez y M. A. García Collazo (eds.). FAO, Ministerio de Agricultura, Facultad de Agronomía (Universidad de Buenos Aires). URL: https://bit.ly/167Xpez.

Garrett, R., E. F. Lambin, and Y. L. P de Waroux. 2017. To eliminate deforestation in South America, reduce differences in regulations across regions and actors. ISID Policy Brief PB-2017-05. Institute for the Study of International Development, McGill University. Montreal, Canada.

Gasparri, N. I., H. R. Grau, and E. Manghi. 2008. Carbon pools and emissions from deforestation in extra-tropical 
forests of northern Argentina between 1900 and 2005. Ecosystems 11(8):1247-1261.

Gasparri, N. I., H. R. Grau, H. R., and J. G. Angonese. 2013. Linkages between soybean and neotropical deforestation: coupling and transient decoupling dynamics in a multi-decadal analysis. Global Environmental Change 23(6):16051614 .

Gautreau, P., L. Langbehn, and L. E. Ruoso. 2014. Movilización de información en el Ordenamiento Territorial de Bosques Nativos de Argentina. La heterogeneidad de los mapeos provinciales y la institucionalización de la problemática ambiental. III Jornadas Nacionales de Investigación y Docencia en Geografía. Tandil, Argentina.

Gibbs, H. K., A. S. Ruesch, F. Achard, M. K. Clayton, P. Holmgren, N. Ramankutty, and J. A. Foley. 2010. Tropical forests were the primary sources of new agricultural land in the 1980s and 1990s. Proceedings of the National Academy of Sciences 107(38):16732-16737.

Giménez, R., J. L. Mercau, J. Houspanossian, and E. G. Jobbágy. 2015. Balancing agricultural and hydrologic risk in farming systems of the Chaco plains. Journal of Arid Environments 123:81-92.

Goldfarb, L., and G. van der Haar. 2016. The moving frontiers of genetically modified soy production: shifts in land control in the Argentinian Chaco. The Journal of Peasant Studies 43(2):562-582.

Graeub, B. E., M. J. Chappell, H. Wittman, S. Ledermann, R. B. Kerr, and B. Gemmill-Herren. 2016. The state of family farms in the world. World Development 87:1-15.

Grau, H. R., N. I. Gasparri, and T. M. Aide. 2005. Agriculture expansion and deforestation in seasonally dry forests of north-west Argentina. Environmental Conservation 32(2):140-148.

Griffiths, N., J. Bono, M. A. García Collazo, and M. Taurian. 2014. Situación de los Ordenamientos Territoriales de Bosques Nativos en la Región Chaqueña - Ley № 26.331. Primer Congreso Internacional de Gran Chaco Americano. Santiago del Estero, Argentina.

Groppo, P. 2014. El sistema territorial y los actores: Mecanismos de participación y negociación en procesos de ordenamiento territorial. En: J. M. Paruelo, E. G. Jobbágy, P. Laterra, H. Diéguez and M. A. García Collazo (eds.). Ordenamiento Territorial: Conceptos, Metodologías y Experiencia. FAO, Ministerio de Agricultura, Facultad de Agronomía (Universidad de Buenos Aires). URL: bit.ly/167Xpez.

Hansen, M. C., P. V. Potapov, R. Moore, M. Hancher, S. Turubanova, A. Tyukavina, D. Thau, et al. 2013. High-resolution global maps of 21st-century forest cover change. Science 342(6160):850-853.

Harvey, D. 2004. The "new imperialism": Accumulation by dispossession. Actuel Marx 35(1):71-90.

Hoekstra, J. M., T. M. Boucher, T. H. Ricketts, and C. Roberts. 2005. Confronting a biome crisis: global disparities of habitat loss and protection. Ecology Letters 8(1):23-29.

INTA. 2015. Guía de prácticas recomendables para sistemas silvopastoriles en Santiago del Estero. Santiago del Estero, Argentina

Jobbágy, E. G., M. D. Nosetto, C. S. Santoni, and G. Baldi. 2008. El desafío ecohidrológico de las transiciones entre sistemas leñosos y herbáceos en la llanura Chaco-Pampeana. Ecología Austral 18(3):305-322.

Lambin, E. F., P. Meyfroidt, X. Rueda, A. Blackman, J. Börner, P. O. Cerutti, et al. 2014. Effectiveness and synergies of policy instruments for land use governance in tropical regions. Global Environmental Change 28:129-140.

Lambin, E. F., H. K. Gibbs, L. Ferreira, R. Grau, P. Mayaux, P. Meyfroidt, et al. 2013. Estimating the world's potentially available cropland using a bottom-up approach. Global Environmental Change 23(5):892-901.

Langbehn, L. 2015 Arenas de conflicto y construcción de problemas públicos ambientales. Un análisis de la productividad del caso de la Ley de Bosques y del Ordenamiento Territorial de Bosques Nativos de Salta (2004-2009). Doctor en Ciencias Sociales. Facultad de Ciencias Sociales, Universidad de Buenos Aires, Argentina.

Langbehn, L. 2016. Arenas públicas, modelos de desarrollo y políticas de protección del ambiente: la Ley de Bosques entre 'conservación' y'producción'. Pp. 141-168 en G. Merlinsky (ed.). Cartografías del conflicto ambiental en Argentina II. Editorial Ciccus, Buenos Aires, Argentina.

León, C., N. Prudkin, and C. Reboratti. 1985. El conflicto entre producción, sociedad y medio ambiente: la expansión agrícola en el sur de Salta. Desarrollo Económico 25(99):399-420.

Ley 25.3675. 2002. Ley General del Ambiente № 25.675. Primera sección. Argentina. Pp. 3. URL: bit.ly/2M9ZRXp. [Consulta: 30 de abril de 2016].

Ley 26.331. 2009. Ley de Protección ambiental de Bosques Nativos № 26.331. Primera sección. Argentina. Pp. 3. URL: bit.ly/2JSL0DW. [Consulta: 10 de abril de 2016].

Macchi, L., H. R. Grau, P. V. Zelaya, and S. Marinaro. 2013. Trade-offs between land use intensity and avian biodiversity in the dry Chaco of Argentina: A tale of two gradients. Agriculture, Ecosystems and Environment 174:11-20.

Mastrangelo, M. E., and M. C. Gavin. 2014. Impacts of agricultural intensification on avian richness at multiple scales in Dry Chaco forests. Biological Conservation 179:63-71.

Merlinsky, G. 2013 Política, derechos y justicia ambiental. El conflicto del Riachuelo. Fondo de Cultura Económica (FCE), Buenos Aires, Argentina

Ministro de Ambiente y Desarrollo Sustentable de la Nación (MAyDS). 2016. Informe de estado de implementación Ordenamiento Territorial de Bosques Nativos y planes alcanzados por el Fondo Nacional para la Enriquecimiento y la Conservación de los Bosques Nativos. Argentina.

Mioni, W., G. Godoy Garraza, and L. Alcoba. 2013. Tierra sin Mal: Aspectos jurídicos e institucionales del acceso a la tierra en Salta. Ediciones INTA, Buenos Aires, Argentina.

Myers, N., R. A. Mittermeier, C. G. Mittermeier, G. A. Da Fonseca, and J. Kent. 2000. Biodiversity hotspots for conservation priorities. Nature, 403(6772):853.

Nolte, C., Y. L. P. de Waroux, J. Munger, J., T. N. Reis, and E. F. Lambin. 2017. Conditions influencing the adoption of effective anti-deforestation policies in South America's commodity frontiers. Global Environmental Change 43: 
$1-14$.

Nolte, C., B. Gobbi, Y. L. P. de Waroux, M. Piquer-Rodríguez, V. Butsic, and E. F. Lambin. 2017. Decentralized Land Use Zoning Reduces Large-scale Deforestation in a Major Agricultural Frontier. Ecological Economics 136:30-40.

Paruelo, J. M., E. G. Jobbágy, P. Laterra, H. Diéguez, and M. A García Collazo. 2014. Ordenamiento Territorial: Conceptos, Metodologías y Experiencias. FAO, Ministerio de Agricultura, Facultad de Agronomía (Universidad de Buenos Aires). URL: bit.ly/167Xpez.

Paruelo, J. M. 2016. El papel de la Ciencia en el proceso de Ordenamiento Territorial (y en otras cuestiones vinculadas con problemas ambientales). Ecología Austral 26(1):51-58

Paruelo, J. M., M. Texeira, L. Staiano, M. Mastrangelo, M. L. Amdan, and F. Gallego. 2016. An integrative index of Ecosystem Services provision based on remotely sensed data. Ecological Indicators 71:145-154.

Periago, M. E., V. Chillo, and R.A. Ojeda. 2015. Loss of mammalian species from the South American Gran Chaco: empty savanna syndrome? Mammal Review 45(1):41-53.

Piquer-Rodríguez, M., S. Torella, G. Gavier-Pizarro, J. N. Volante, D. Somma, R. Ginzburg, and T. Kuemmerle. 2015. Effects of past and future land conversions on forest connectivity in the Argentine Chaco. Landscape Ecology 30(5): 817-833.

Quiroga, V. A., G. I. Boaglio, A. J. Noss, and M. S. Di Bitetti. 2014. Critical population status of the jaguar Panthera onca in the Argentine Chaco: camera-trap surveys suggest recent collapse and imminent regional extinction. Oryx 48(1): 141-148.

Quiroga, V. A., A. J. Noss, A. Paviolo, G. I. Boaglio, and M. S. Di Bitetti. 2016. Puma density, habitat use and conflict with humans in the Argentine Chaco. Journal for Nature Conservation 31:9-15.

Reboratti, C. 2008. Desarrollo agropecuario, ambiente y población rural. En Agro y Ambiente: una agenda compartida para el desarrollo sustentable. Foro de la Cadena Agroindustrial Argentina

Red Agroforestal Chaco Argentina (REDAF). 2012. Monitoreo de Deforestación de los Bosques Nativos en la Región Chaqueña Argentina. Informe No 1: Ley de Bosques, análisis de deforestación y situación del Bosque chaqueño en la provincia de Salta. Reconquista, Santa Fe. Pp. 34. URL: bit.ly/2MG6Tnybit.ly/2I6APpw. [Consulta: 15 de junio 2014].

Red Agroforestal Chaco Argentina (REDAF). 2013. Tercer informe de Conflictos sobre tenencia de tierra y ambientales en la región del chaco argentino. $3^{\circ}$ Informe. Reconquista, Santa Fe. Pp. 98.

Red Agroforestal Chaco Argentina (REDAF). 2016. Para "recomponer" 11 mil hectáreas desmontadas, demandan a un finquero por 171 millones de pesos. URL: bit.ly/2MJgutV. [Consulta: 20 de agosto 2017].

Roux, D., K. Rogers, H. Biggs, P. Ashton, and A. Sergeant, A. 2006. Bridging the science-management divide: moving from unidirectional knowledge transfer to knowledge interfacing and sharing. Ecology and Society 11(1):4.

Rudel, T. K., and R. Meyfroidt. 2014. Organizing anarchy: The food security-biodiversity-climate crisis and the genesis of rural land use planning in the developing world. Land Use Policy 36:239-247.

Schmidt, M., and L. Langbehn. 2016. La "Ley de Bosques" en Argentina, entre la regulación de la deforestación y la continuidad de los conflictos territoriales. VI Congreso Argentino y Latinoamericano de Antropología Rural (CALAAR). Salta, Argentina.

Schmidt, M. 2012. Situación de la tierra en la provincia de Salta. Una aproximación al contexto previo al Ordenamiento Territorial de Bosques Nativos. Estudios Rurales 1:75-103.

Schmidt, M. 2013. Crónicas de un (Des)Ordenamiento Territorial. Disputas por el territorio, modelos de desarrollo y mercantilización de la naturaleza en el este salteño. Doctor en Ciencias Sociales. Facultad de Ciencias Sociales, Universidad de Buenos Aires, Argentina.

Schmidt, M. 2014. Ordenadores y ordenados. Actores en disputa en el Ordenamiento Territorial de Bosques Nativos en la provincia de Salta. Cuadernos de Antropología 11:37-55.

Seghezzo, L., J. N. Volante, J. M. Paruelo, D. J. Somma, E. C. Buliubasich, H. E. Rodríguez, and M. Hufty. 2011. Native Forests and Agriculture in Salta (Argentina) Conflicting Visions of Development. The Journal of Environment and Development 20(3):251-277.

Seghezzo, L., C. Venencia, E. C. Buliubasich, M. A. Iribarnegaray, and J. N. Volante. 2017. Participatory, Multi-Criteria Evaluation Methods as a Means to Increase the Legitimacy and Sustainability of Land Use Planning Processes. The Case of the Chaco Region in Salta, Argentina. Environmental Management 59(2):307-324.

Silvetti, F., G. Soto, D. M. Cáceres, and D. Cabrol. 2013. ¿Por qué la legislación no protege los bosques nativos de Argentina?: Conflictos socioambientales y políticas públicas. Mundo Agrario 13(26). URL: bit.ly/2tdlBuq.

Unidad de Manejo del Sistema de Evaluación Forestal (UMSEF). 2016. Monitoreo de la superficie de bosque nativo de la República Argentina. Regiones Forestales Parque Chaqueño, Yungas, Selva Paranaense y Espinal. Dirección de Bosques, Ministerio de Ambiente y Desarrollo Sustentable. Argentina

Vallejos, M., J. N. Volante, M. J. Mosciaro, L. Vale, M. L, Bustamante, and J. M. Paruelo. 2015. Transformation dynamics of the natural cover in the Dry Chaco ecoregion: a plot level geo-database from 1976 to 2012. Journal of Arid Environments 123:3-11.

Vakis, R., J. Rigolini, and L. Lucchetti. 2016. Left behind: chronic poverty in Latin America and the Caribbean. World Bank Publications, Washington D.C., Estados Unidos.

Volante, J. N., and L. Seghezzo. 2018. Can't see the forest for the trees: Can declining deforestation trends in the Argentinean Chaco Region be ascribed to efficient law enforcement? Ecological Economics 146(2018):408-413.

West, P. C., H. K. Gibbs, C. Monfreda, J. Wagner, C. C. Barford, S. R. Carpenter, and J. A. Foley. 2010. Trading carbon for food: Global comparison of carbon stocks vs. crop yields on agricultural land. Proceedings of the National Academy of Sciences 107(46):19645-19648. 